\title{
Peptide 946-Tetanus Peptide Conjugate Melanoma Vaccine/QS21
}

National Cancer Institute

\section{Source}

National Cancer Institute. Peptide 946-Tetanus Peptide Conjugate Melanoma

VaccinelQS21. NCI Thesaurus. Code C2469.

A vaccine comprised of peptide 946 , a gp100 peptide recognized by melanoma-specific cytotoxic T lymphocytes, conjug ated with a modified T-helper epitope from tetanus toxoid and emulsified with QS21, a saponin vaccine adjuvant. (NCI) 